\title{
PIK3CA mutations in androgen receptor-positive triple negative breast cancer confer sensitivity to the combination of $\mathrm{PI} 3 \mathrm{~K}$ and androgen receptor inhibitors
}

Brian D Lehmann", Joshua A Bauer', Johanna M Schafer ${ }^{1}$, Christopher S Pendleton ${ }^{1}$, Luojia Tang ${ }^{1}$, Kimberly C Johnson ${ }^{1}$, Xi Chen², Justin M Balko ${ }^{3}$, Henry Gómez ${ }^{4}$, Carlos L Arteaga ${ }^{3}$, Gordon B Mills ${ }^{5}$, Melinda E Sanders ${ }^{6}$ and Jennifer A Pietenpol ${ }^{1,7^{*}}$

\begin{abstract}
Introduction: Triple negative breast cancer (TNBC) is a heterogeneous collection of biologically diverse cancers, which contributes to variable clinical outcomes. Previously, we identified a TNBC subtype that has a luminal phenotype and expresses the androgen receptor (AR+). TNBC cells derived from these luminal AR + tumors have high frequency phosphatidylinositol-4,5-bisphosphate 3-kinase catalytic subunit alpha (PIK3CA) mutations. The purpose of this study was to determine if targeting phosphoinositide 3-kinase (PI3K) alone or in combination with an AR antagonist is effective in AR + TNBC.

Methods: We determined the frequency of activating PIK3CA mutations in AR + and AR- TNBC clinical cases. Using AR + TNBC cell line and xenograft models we evaluated the effectiveness of PI3K inhibitors, used alone or in combination with an AR antagonist, on tumor cell growth and viability.

Results: PIK3CA kinase mutations were highly clonal, more frequent in AR+vs. AR- TNBC (40\% vs. 4\%), and often associated with concurrent amplification of the PIK3CA locus. PI3K/mTOR inhibitors had an additive growth inhibitory effect when combined with genetic or pharmacological AR targeting in AR + TNBC cells. We also analyzed the combination of bicalutamide +/- the pan-PI3K inhibitor GDC-0941 or the dual PI3K/mTOR inhibitor GDC-0980 in xenograft tumor studies and observed additive effects.
\end{abstract}

Conclusions: While approximately one third of TNBC patients respond to neoadjuvant/adjuvant chemotherapy, recent studies have shown that patients with $A R+T N B C$ are far less likely to benefit from the current standard of care chemotherapy regimens and novel targeted approaches need to be investigated. In this study, we show that activating PIK3CA mutations are enriched in AR + TNBC; and, we show that the growth and viability of AR + TNBC cell line models is significantly reduced after treatment with PI3K inhibitors used in combination with an AR antagonist. These results provide rationale for pre-selection of TNBC patients with a biomarker (AR expression) to investigate the use of AR antagonists in combination with PI3K/mTOR inhibitors.

\footnotetext{
* Correspondence: j.pietenpol@vanderbilt.edu

'Department of Biochemistry, Vanderbilt-Ingram Cancer Center, Vanderbilt

University School of Medicine, Preston Research Building, 2200 Pierce

Avenue, Nashville, TN 37232, USA

7Vanderbilt-Ingram Cancer Center, 652 Preston Research Building, Nashville,

TN 37232, USA

Full list of author information is available at the end of the article
}

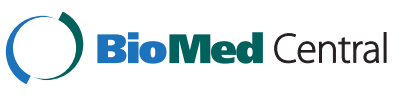

(c) 2014 Lehmann et al.; licensee BioMed Central Ltd. This is an Open Access article distributed under the terms of the Creative Commons Attribution License (http://creativecommons.org/licenses/by/4.0), which permits unrestricted use, distribution, and reproduction in any medium, provided the original work is properly credited. The Creative Commons Public Domain Dedication waiver (http://creativecommons.org/publicdomain/zero/1.0/) applies to the data made available in this article, unless otherwise stated. 


\section{Introduction}

Over the past decade, the term triple-negative breast cancer (TNBC) has been used to classify tumors that lack detectable expression of the estrogen receptor (ER) and progesterone receptor (PR) and amplification of human epithelial growth factor receptor 2 (HER2). TNBC tumors are generally more aggressive than their ER+ counterparts, with higher rates of relapse in the early stages and decreased overall survival in the metastatic setting [1,2]. Although successful targeted therapies exist for $\mathrm{ER}+$ and HER2-amplified breast cancer, TNBC has been particularly difficult to treat given the biology of the disease has not been well understood. TNBC represents multiple independent subtypes likely requiring different therapeutic approaches, and until recently, targets for therapeutic intervention have remained elusive [3]. Current standard of care for TNBC consists of treating patients with a combination of anthracyclines and taxanes and is based on the positive results of numerous trials showing that chemotherapy combinations with these drugs, in the neo-adjuvant setting in particular, can give significant increased clinical response rates [4]. Nonetheless, there is a major need for new therapeutic options for patients suffering from TNBC.

Investigators exploring the genomic architecture of TNBCs discovered a spectrum of somatic mutations; however, only a few loci are recurrently mutated with significant frequency $[5,6]$. TP53 mutations are the most frequent clonal events (62\%) followed by mutations in PIK3CA (10.2\%), the gene that encodes the p110 $\alpha$ catalytic subunit of phosphatidylinositol-3 kinase (PI3K). Through integrated analyses of numerous world-wide gene expression (GE) datasets and a panel of TNBC lines, our laboratory provided insight into the heterogeneity of TNBC disease by identifying distinct molecular subtypes displaying unique biology that includes two basal-like (BL1 and BL2), an immunomodulatory (IM), a mesenchymal (M), a mesenchymal stem-like (MSL), and a luminal androgen receptor (LAR) subtype [3]. Of note, we demonstrated that LAR cells are in part dependent on AR signaling as siRNA-mediated AR knockdown or pharmacological inhibition of AR by bicalutamide (CDX) greatly decreases cell viability and tumor growth [3]. Also, we observed that all commercially available AR-positive (AR+) TNBC cell lines contain the PIK3CA mutation (H1047R) and are highly sensitive to the PI3K/mTOR inhibitor NVP-BEZ235 [3]. Collectively, these findings are consistent with observations that hormonally responsive cancers, such as those expressing ER [7] and AR [5,8] are more likely to acquire PIK3CA mutations, thus prompting the experiments and discoveries reported herein.

With the goal of generating pre-clinical data that could be advanced to clinical trial design for the various subtypes of TNBC, we further investigated the molecular features of the AR + TNBC subtype, LAR. First, we discovered that PIK3CA kinase domain mutations are a frequent event in $\mathrm{AR}+\mathrm{TNBC}$ clinical cases. We found that genetic or pharmacological targeting of AR in LAR cells increases the growth inhibitory activity of PI3K inhibitors. Further, we explored the combination of AR antagonism and PI3K inhibition and found an additive or synergistic effect on AR + TNBC cell growth. Given that AR protein expression is a reliable surrogate of LAR gene expression within TNBC, our preclinical findings provide a strong rationale for the use of $\mathrm{AR}$ as a biomarker for the selection of TNBC patients for clinical trials, which would investigate the efficacy of therapeutic combinations that simultaneously target AR and PI3K.

\section{Methods}

\section{Acquisition of tumor tissue for this study}

After the Vanderbilt University Institutional Review Board (IRB) approved the project protocol and waived the need for patient consent, de-identified TNBC tumor tissues were obtained. ER, PR and HER2 status as determined at the time of clinical evaluation was available for all specimens and was retested for the purposes of this project (see Immunostaining section below). Both frozen and formalin-fixed paraffin-embedded (FFPE) tissue samples were obtained. In addition, surgically resected tumor samples were obtained from patients with TNBC diagnosed and treated with neoadjuvant chemotherapy at the Instituto Nacional de Enfermedades Neoplásicas in Lima, Perú. Clinical and pathological data were retrieved from medical records under an institutionally approved protocol (INEN 10-018) and de-identified prior to distribution to Vanderbilt University Medical Center. Tumors were determined as triple-negative if they were negative for ER, PR and HER2 overexpression measured by immunohistochemistry (IHC).

\section{Immunostaining}

Both frozen sections and FFPE tissue were used for immunohistochemical studies. For FFPE sections, antigen retrieval for both ER and PR were performed using citrate buffer ( $\mathrm{pH} \mathrm{6)}$ in a decloaking chamber (Biocare Medical, Concord, CA, USA). ER (6 F11, Vector Labs, Burlingame, CA) PR (PgR636; DAKO, Carpinteria, CA) and HER2 (\#2242; Cell Signaling, Danvers, MA) antibodies were utilized at 1:200, 1:50, and 1:200 dilutions, respectively. Antigen retrieval was omitted for the frozen tissues and the antibody dilutions were decreased to 1:400, 1:100 and 1:400 respectively. Visualization for both antibodies was performed using the Envision Detection System (DAKO) and 3,3diaminobenzidine (DAB) (DAKO) as the chromogen. The percentage of invasive tumor cells with nuclear ER and PR staining and the average intensity of all positively staining tumor cells in the section were manually counted 
as per College of American Pathologists/American Society of Clinical Oncology (CAP/ASCO) guidelines [9]. The percentage of invasive tumor cells with membranous HER2 staining at the highest intensity level was manually assessed and recorded as per CAP/ASCO guidelines [10].

For FFPE sections, antigen retrieval for AR (DAKO, clone AR411) was performed using citrate buffer $\mathrm{pH} 6.0$ in a decloaking chamber followed by 1-h incubation with antibody at room temperature at a dilution of 1:300. Antigen retrieval for p-AKT p473 (Cell Signaling) was performed using Trilogy buffer $\mathrm{pH} 6.0$ (Cell Marque) in a decloaking chamber (Biocare Medical) followed by overnight incubation at $4^{\circ}$ at a 1:25 antibody dilution. Antigen retrieval was omitted for the frozen tissues and the antibody dilutions were decreased to 1:600 and 1:30, respectively. To reduce the possibility of false negatives, tumors were determined to be AR + when $>30 \%$ of the cells showed nuclear positivity of any detectable intensity level.

\section{Fluorescence in situ hybridization for HER2}

Both frozen sections and FFPE tissue were used for fluorescence in situ hybridization (FISH). FISH for detection of possible amplification of HER2 was performed using the PathVysion HER-2 DNA Probe Kit (PathVysion Kit, Abbott Molecular, Des Planes, IL, USA) utilizing the Vysis LSI HER2/neu 17q11.2-12 SpectrumOrange ${ }^{\text {mu }}$ and Vysis CEP 17 17p11.1-q11.1 SpectrumGreen Alpha Satellite DNA probes for cases with $2+$ or $3+$ expression by IHC. Images were visualized on a Fluorescence Olympus BX60 Microscope and analyzed using the Genus ${ }^{\mathrm{Tw}}$ for Genetic Image Analysis software, version 3.6. The ratio of HER2 to CEP 17 signals was recorded and reported as an average ratio as per CAP/ASCO guidelines [10].

\section{PIK3CA mutation evaluation}

Sanger sequencing was used to detect mutations in exons 9 and 20 of PIK3CA of PCR-amplified regions from genomic DNA. Genomic DNA was isolated from FFPE tissues of 25 $\mathrm{AR}+$ and 25 AR- TNBC tumors (QiaAmp FFPE, Qiagen, Valencia, CA). Genomic DNA (20 ng) was PCR-amplified in exons 9 (168 bp fragment) and 20 (159 bp fragment) using the following amplification primers: PIK3CA_ex9_F: GTTTTCCCAGTCACGACGGAAAATGACAAAGAACA GC; PIK3CA_ex9_R: CAGGAAACAGCTATGACCTGAG ATCAGCCAAATTCA; PIK3CA_ex20_F: GTT TTCCCA GTCACGACGGAATGCCAGAACTACAATCTTTT and PIK3CA_ex20_R: CAGGAAACAGCTATGACTGTGTGG AAGATCCAATCCA.

\section{Subtyping TNBC cases and validating PIK3CA mutations in the The Cancer Genome Atlas (TCGA)}

RNA-seq (lvl 3), somatic mutations (lvl3) and reversephase protein array (RPPA, lvl3) data for breast cancer were downloaded from TGCA (tcga-data.nci.nih.gov/). RNA-seq data (RPKM) was combined and molecularly subtyped using the online TNBCtype software according to published methods [11]. After removal of potential ER + samples, 102 TNBC samples were identified and assigned a molecular subtype, which included four ER + samples by IHC that were called ER- by RNA (Additional file 1: Table S1). Differences in RNA, protein and mutations were evaluated by Student's $t$-test.

\section{Reverse-phase protein array on TNBC cell lines}

Tumor or cell lysates were two-fold serial diluted for five dilutions (from undiluted to 1:16 dilution) and arrayed on a nitrocellulose-coated slide in $11 \times 11$ format. Samples were probed with antibodies by the Catalyzed Signal Amplification (DAKO, Carpinteria, CA) amplification approach and visualized by $\mathrm{DAB}$ colorimetric reaction. Slides were scanned and density quantified by MicroVigene. Relative protein levels for each sample were determined by interpolation of each dilution curve from the standard curve (supercurve) of the slide (antibody) as previously described [12]. These values were log2-transformed and normalized for protein loading by linear transformation. Linear normalized data were then median-centered for heatmap comparisons.

\section{Cell proliferation/viability assays and inhibitory concentration of $50 \%$ (EC50) determination}

All cell lines were maintained in culture as previously described [3]. To evaluate response to dihydrotestosterone (DHT) cells were grown in charcoal-stripped (CS) media for two days prior to plating in 96-well tissue culture dishes. Media were then changed to fresh CSmedia alone or increasing doses of DHT and viability determined by measuring fluorescent intensity after metabolic reduction of AlamarBlue five days later. For drug treatments, breast cancer cell lines and HMECs were seeded (3,000 to 10,000 cells) in quadruplicate wells in 96-well plates. After attachment, media was replaced with either fresh media (control) or media containing half-log serial dilutions of the following drugs: GDC-0941 (10 nM-3000 nM), GDC0980 (1 nM-300 nM), BKM120 (10 nM-3000 nM) and NVP-BEZ235 (1 nM-300 nM) purchased from Selleck Chemicals (Houston, TX) or in combination with bicalutamide (CDX) (Sigma, St. Louis, MO). Viability was determined by measuring fluorescent intensity after metabolic reduction of AlamarBlue in the presence/absence of drug incubation for $72 \mathrm{~h}$. Viability assays were performed in triplicate and replicates were normalized to untreated wells. EC50 values were determined after double log-transformation of dose response curves as previously described [3]. 


\section{shRNA knockdown of AR}

We transfected 293FT cells with Lipofectamine 2000 (Invitrogen, Grand Island, NY) the packaging vectors pPAX2 and pMD2.g (Addgene, Cambridge, MA) along with pLKO.1-puro Misson shRNA constructs (Sigma) targeting AR or a non-targeting control. Viral media were harvested $48 \mathrm{~h}$ post transfection and added to target cells along with polybrene $(10 \mu \mathrm{g} / \mathrm{mL})$. At $72 \mathrm{~h}$ post infection MDA-MB-453 (8,000 cells/well), CAL-148 (5,000 cells/well), SUM-185 (8,000 cells/well) and MFM$223(7,000$ cells/well) were seeded in quadruplicate in 96-well plates. After attachment, media were replaced with fresh media (control) or media containing half-log dilutions of (BKM120 10 to 3,000 nM, GDC-0941 10 to 3,000 nM, NVP-BEZ235 1 to $300 \mathrm{nM}$ and GDC0980 3 to $1,000 \mathrm{nM})$. After incubation in drug for $72 \mathrm{~h}$, viability was measured with AlamarBlue as previously described [3].

\section{Forced suspension viability assay}

CAL-148, MFM-223, MDA-MB-453 and SUM-185 cells were plated (10,000 cells/well) in quadruplicate into 96-well plates coated with $0.9 \%$ agarose diluted in corresponding media. Following five days of growth, cells were treated with increasing half-log concentrations of GDC-0941 (10 to 3,000 nM), GDC0980 (1 nM to 300 $\mathrm{nM})$, BKM120 (10 nM to 3,000 nM) and NVP-BEZ235 $(1 \mathrm{nM}$ to $300 \mathrm{nM})$ alone or in combination with $25 \mu \mathrm{M}$ $\mathrm{CDX}$ for an additional $72 \mathrm{~h}$, after which cells were imaged and viability determined by AlamarBlue.

\section{Immunoblotting}

Cells were trypsinized, lysed and protein concentration was determined by immunoblot as previously described (12) with the following antibodies; AR polyclonal antibody, (SC-N20, Santa Cruz Biotechnology, Dallas, Texas), glyceraldehyde-3-phosphate dehydrogenase (GAPDH) (EMD Millipore, Billerica, MA) and the following antibodies from Cell Signaling pS6-Ser235/236 (\#4856), S6 (\#2317), p-AKT-S473 (\#9271), and AKT (\#9272).

\section{Xenograft tumor studies}

Five-week-old female athymic nude Foxn $1^{\text {nu }}$ mice (Harlan, Indianapolis, IN) were injected (subcutaneously) with either approximately $4 \times 10^{6}$ (CAL-148) cells suspended in media $(200 \mu \mathrm{L})$ or approximately $10 \times 10^{6}$ (MDA-MB453) cells suspended in $100 \mu \mathrm{L}$ media and $100 \mu \mathrm{L}$ matrigel into each flank using a 22-gauge needle. Once tumors reached a volume of 25 to $50 \mathrm{~mm}^{3}$, mice were randomly assigned to treatment or vehicle arms. Treatments included CDX per os (p.o.) (100 mg/kg/d) or GDC0980 per os (p.o.) $(7.5 \mathrm{mg} / \mathrm{kg} / \mathrm{d})$ in $5 \%$ dimethyl sulfoxide (DMSO), $0.5 \%$ carboxymethyl cellulose (MCT) or NVP-BEZ235 per os (p.o.) $(50 \mathrm{mg} / \mathrm{kg} / \mathrm{d})$ in $0.5 \% \mathrm{MCT}$ as well as the combination of CDX and either GDC-0980 or NVPBEZ235 according to the above concentrations. Tumor diameters were serially measured at the indicated times with digital calipers and tumor volumes were calculated as width $^{2} \times$ length $/ 2$. All studies were assessed for the ethical use and treatment of animals and received ethical approval by the Institutional Animal Care and Use Committee of Vanderbilt University.

\section{Results}

AR + TNBCs are enriched for PIK3CA activating mutations

Previously we demonstrated high frequency of PIK3CA mutations in AR + TNBC cell lines [3]. To determine if PIK3CA mutations frequently arise in AR + TNBC, we performed Sanger sequencing on $26 \mathrm{AR}+$ and $26 \mathrm{AR}$ TNBC clinical cases. We sequenced PCR-amplified regions from exons 9 and 20, the region of the gene harboring the most frequently occurring activating mutations. Consistent with our observations and ruling out in vitro selection for PIK3CA mutations in AR + LAR cell lines, we found that PIK3CA mutations were significantly $(P<0.002)$ enriched in $\mathrm{AR}+(10 / 25,40 \%)$ versus ARTNBCs $(1 / 25,4 \%)$ (Figure 1A). Nearly all of the detected mutations occurred in the kinase domain (H1047R/L; 9 of 10) with only one occurring at E545K in the helical domain.

\section{Validation of PIK3CA mutations in AR-expressing TNBC}

To verify the high frequency of PIK3CA mutations in TNBC tumors with elevated levels of AR protein, we analyzed the RNA-seq, DNA-seq and reverse-phase protein array (RPPA) data in TCGA breast cancer cohort [6]. In order to remove potential false negatives for ER, we calculated the percentile of ER gene expression for each tumor specimen and removed tumors that displayed ER expression above the 75th percentile of all genes. The resulting 102 tumors were classified according to TNBC molecular subtypes using the TNBCtype algorithm tool (Additional file 1: Table S1) [11]. The distribution of samples across the TNBC subtypes was similar to that which we had previously reported [3,11], (UNS $=9(8.8 \%)$, $\mathrm{BL} 1=22(21.6 \%), \mathrm{BL} 2=8$ (7.8\%), $\mathrm{IM}=18(17.6 \%), \mathrm{M}=24$ $(23.5 \%), \mathrm{MSL}=8(7.8 \%)$ and $\mathrm{LAR}=13(12.7 \%)$; Figure 1B and Additional file 1: Table S1). As expected, analysis of RNA-seq data generated from the LAR subtype samples confirmed significant levels of AR RNA $(1,219$ versus 101 reads per kilobase per million reads (RPKM), $P<0.0001)$ and protein by RPPA $(0.64$ versus -1.15 , $P<0.0001$ ) (Figure 1B and Additional file 1: Table S1). PIK3CA mutations were relatively infrequent (10 of 102, 9.8\%) in TNBC cases overall in TCGA dataset (Figure 1B). However, when sorted by subtype, PIK3CA mutations were significantly enriched among LAR TNBCs (6 of 13, 46.2\%) compared to all other TNBC subtypes (4 of 89 , 
A

\begin{tabular}{|c|c|c|c|c|c|}
\hline \multicolumn{6}{|c|}{ Table 1. PIK3CA mutations in AR expressing TNBC } \\
\hline \multirow{2}{*}{ TNBC } & \multirow{2}{*}{$\mathrm{N}=$} & \multicolumn{5}{|c|}{ PIK3CA Mutations } \\
\cline { 3 - 6 } & & H1047R & H1047L & E545K & Mutations \\
\hline AR+ & 25 & $\begin{array}{c}8 \\
(32 \%)\end{array}$ & $\begin{array}{c}1 \\
(4 \%)\end{array}$ & $\begin{array}{c}1 \\
(4 \%)\end{array}$ & $\begin{array}{c}10 \\
(40 \%)\end{array}$ \\
\hline AR- & 25 & $\begin{array}{c}1 \\
(4 \%)\end{array}$ & $\begin{array}{c}0 \\
(0 \%)\end{array}$ & $\begin{array}{c}0 \\
(0 \%)\end{array}$ & $\begin{array}{c}1 \\
(4 \%)\end{array}$ \\
\hline
\end{tabular}

B

TCGA TNBC Tumors

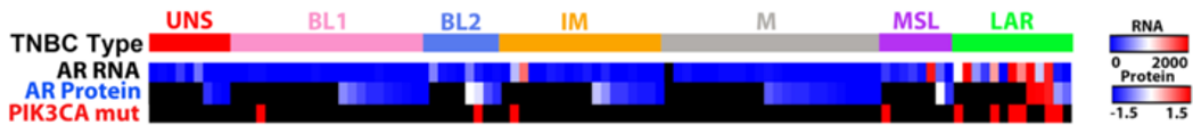

Figure 1 Identification of PIK3CA mutations in androgen receptor (AR) + triple-negative breast cancer (TNBC) cell lines and tumors. (A) Table displays the frequency of PIK3CA mutations (H1047R) in 25 TNBC AR + and 25 AR- TNBC human tumors. (B) Panel displays the TNBC molecular subtypes of The Cancer Genome Atlas (TCGA) breast cancer tumors with corresponding heatmaps showing the relative levels of AR RNA (RNA-seq) and AR protein (reverse-phase protein array (RPPA)). Those cases lacking protein or RNA evaluation are colored in black. The bottom row is a color bar indicating PIK3CA mutations (red) or wild-type (black) within the TCGA TNBC cases.

$4.5 \%, P<0.0001$ ), with a predominance of H1047R mutations, consistent with our findings above (Figure 1B).

We evaluated the allele frequency of mutant and wildtype alleles in three AR + TNBCs containing H1047R PIK3CA. Since DNA-seq data were not available for two samples, we determined if allele frequencies in RNA-seq were comparable to DNA-seq from a tumor sample with both types of data (TCGA-C8-A12L). DNA-seq and RNA-seq (86\% versus $77 \%$ ) produced similar mutant allele frequencies, suggesting that RNA-seq could provide a reliable estimate of allele frequency (Additional file 2: Figure S1A). All three AR+/PIK3CA mutant TNBCs demonstrated a high allele frequency for the H1047R mutation $(77 \%, 67 \%$, and $53 \%)$, greater than the expected frequency for a heterozygous mutation (in a tumor cellularity $>80 \%$ ), suggesting that either the other allele had lower expression or that the mutant allele was amplified (Additional file 2: Figure S1B). We further evaluated the DNA copy number $(\mathrm{CN})$ of $14 \mathrm{MB}$ of DNA sequence surrounding the PIK3CA locus. $\mathrm{CN}$ analysis showed that the PIK3CA locus was amplified (1.544, 1.436, and 1.316, $\log 2)$ in $\mathrm{AR}+/ \mathrm{PIK} 3 \mathrm{CA}$ mutant tumors, including one tumor (TCGA-C8-A12L) with a focal amplification of only 12 genes (Additional file 3: Figure S2). The concurrent mutation and amplification of PIK3CA frequently occur in all of breast cancer with $42 \%$ (18 of 43) of tumors with amplified PIK3CA also harboring a PIK3CA mutation (Additional file 3: Figure S2).

LAR TNBC cell lines stimulated by dihydrotestosterone have increased $\mathrm{PI} 3 \mathrm{~K}$ pathway activity and are sensitive to $\mathrm{PI3K} / \mathrm{TOR}$ pathway inhibitors

To demonstrate that $\mathrm{AR}+\mathrm{TNBC}$ cell lines are responsive ligands, we measured the proliferation of the AR-dependent prostate cancer cell line LNCAP and AR + TNBC cell lines five days after treatment with increasing doses of
Dihydrotestosterone (DHT). Low levels of DHT (femtomolar and picomolar concentrations) were sufficient to increase cell number two-fold in both LNCAP and AR + TNBC cell lines, demonstrating that proliferation of the cell lines can be increased by AR signaling (Figure 2A).

Since the AR + TNBC cell lines have intact AR signaling and PIK3CA mutations, we used RPPA to investigate AR, PTEN, p-AKT (S473 and T308) and p-GSK3 3 (S21/ S9) protein levels in TNBC cell lines. We assessed PI3K pathway activity as determined by relative levels of p-AKT and p-GSK3 $\beta$ across the cell panel and, in particular in AR-expressing, PIK3CA mutant TNBCs (Additional file 4: Table S2). Elevated PI3K signaling correlated with PIK3CA mutation and loss of PTEN in TNBC cell lines as evidenced by high expression of activated p-AKT S473 (2.18 versus $-0.23, P=0.007)$ and $\mathrm{p}$-AKT T308 (1.45 versus $-0.10, P=0.0032)$, and increased levels of $\mathrm{p}$-GSK3 $\beta$ S9 (1.00 versus $-0.26, P=0.0094)$ and $p-G S K 3 \beta$ S21/S9 (1.12 versus $-0.19, P=0.0026$ ) (Figure $2 \mathrm{~B}$ ). Similarly, those cell lines that are null for PTEN (BT549, CAL51, HCC1395, HCC1806, HCC1937, HCC38, HCC70, MDAMB-436 and MDA-MB-468) displayed higher levels of PI3K signaling (p-AKT S473 1.28 versus $-1.28, P=0.0001$, p-AKT T308 0.41 versus $-0.53, P=0.0018, p-G S K 3 \beta$ S9 0.37 versus $-0.76, P=0.0002$ and $p-G S K 3 \beta$ S21/S9 0.26 versus $-0.59, P=0.0009$ ).

To expand on the RPPA data, we performed immunoblot analyses of the PI3K/mTOR pathway in four LAR cell lines (CAL-148, MDA-MB-453, MFM-223, and SUM-185), as well as LNCaP cells (positive control) and primary cultures of human mammary epithelial cells (HMEC; negative control)) (Figure 2C). AR was expressed in the LAR TNBC cell lines at levels equal to or greater than LNCaP cells. With the exception of SUM-185 cells, AR + TNBC cell lines displayed higher levels of p-AKT (S473) and phosphorylated ribosomal S6 than human mammary epithelial 
A
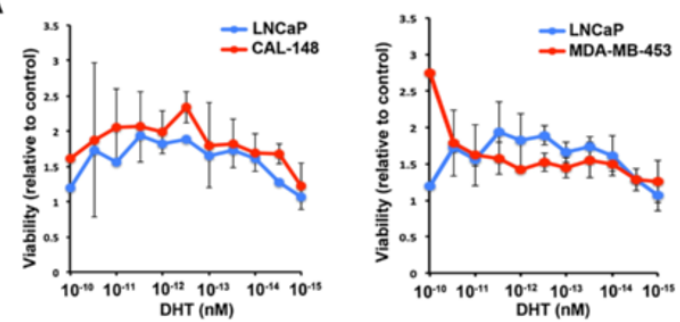

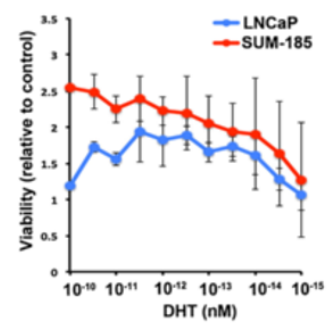

B

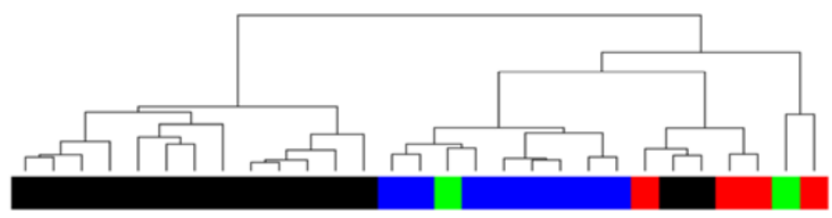

PI3K Pathway Alteration

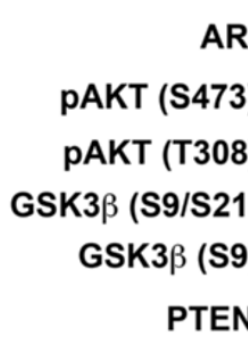

AR

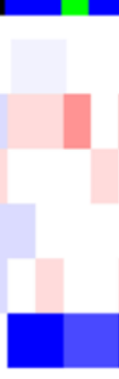

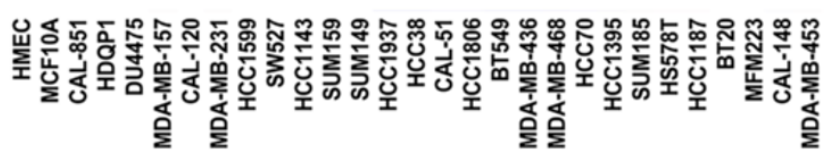

TNBC Cell Lines
PIK3CA mutation

PTEN Deficiency

PIK3CA mut and PTEN Deficiency

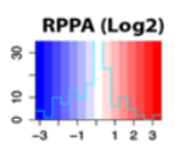

C

D

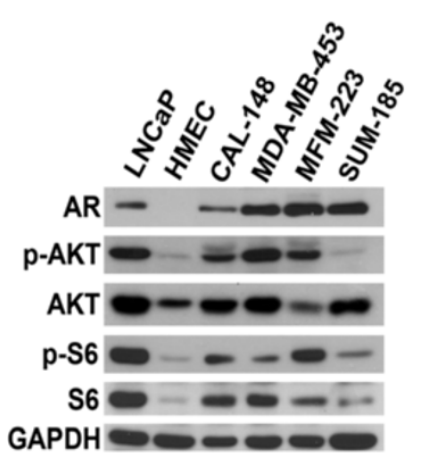

HMEC

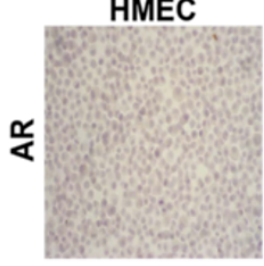

CAL-148

MDA-MB-453

MFM-223

SUM-185

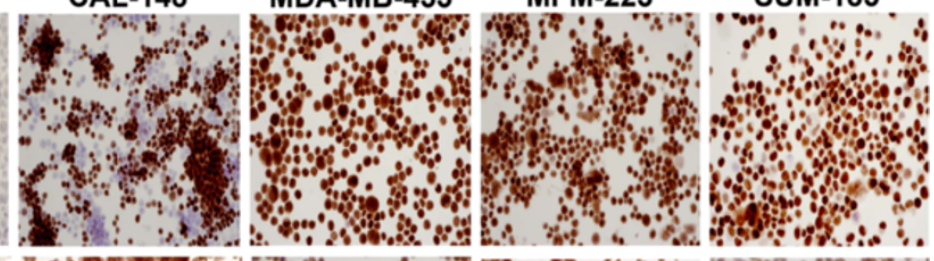

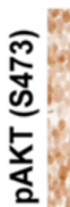

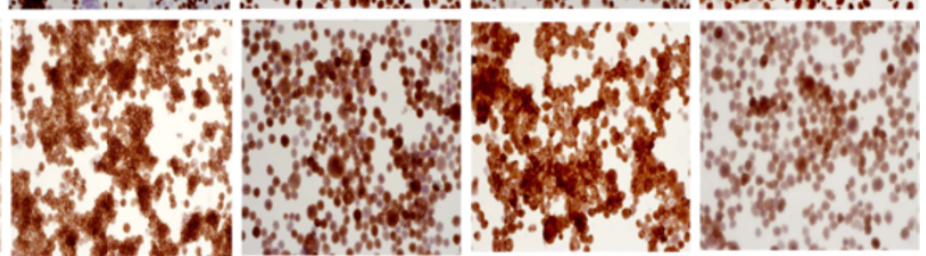

Figure 2 Androgen receptor (AR) + triple-negative breast cancer (TNBC) cell lines have activated PI3K signaling and respond to dihydrotestosterone (DHT) stimulation (A) Graphs display relative viability of the prostate cancer cell line (LNCAP, red line) compared to each of the AR + TNBC cell lines (blue line) 5 days after addition of increasing doses of DHT in charcoal-stripped media. (B) Heatmap displays relative protein levels (reverse-phase protein array, RPPA) of AR, p-AKT (S473 and T308), p-GSK3 $\beta$ (S9 and S21) and PTEN across indicated TNBC cell lines. Unsupervised hierarchical clustering was performed on PI3K pathway proteins (PTEN, p-AKT and p-GSK3 3 ). Known PI3K pathway aberrations are indicated in the colorbar as PTEN loss (blue) or PIK3CA mutations (red). (C) Immunoblot displays relative protein levels of AR, p-AKT, p-S6 in AR-expressing prostate cancer (LNCaP), primary cultures of human mammary epithelial cells (HMECs) and the indicated LAR TNBC cell lines. Glyceraldehyde-3-phosphate dehydrogenase (GAPDH) serves as a loading control. (D) Immunohistochemistry of indicated proteins was performed on cell lines to assess levels of AR and P-AKT (S473). Results are representative of three independent experiments. 
cells (HMECs), suggesting activation of PI3K and downstream mTOR, respectively (Figure $2 \mathrm{C}$ ).

To evaluate the uniformity of AR expression, we performed IHC for AR and p-AKT (S473) on LAR cell lines (Figure 2D). The AR-expressing cell lines displayed differing percentages of cells positive for AR protein expression (CAL-148: approximately 60\%, MDA-MB-453: $>95 \%$, MFM-223: >95\% and SUM-185: >95\%). The lower percentage of AR + cells in the CAL-148 cell line is consistent with decreased levels by immunoblot analysis. Furthermore, the IHC analysis for p-AKT had similar trends to SUM-185 and HMECs, having low levels in both IHC and immunoblot analysis (Figure 2D). To determine if $\mathrm{AR}+$ cells displayed activated AKT, we performed dual immunofluorescence staining for AR and p-AKT (Additional file 5: Figures S3 and Additional file 6: Figure S4). The localization of p-AKT was primarily cytoplasmic, however due to the cytospin preparation and lack of adherence, localization is atypical. Regardless, nearly all AR + cells stained positive for p-AKT. Since there was heterogeneity in AR expression, we performed fluorescence-activated cell sorting (FACS) of AR in the AR + cell lines. SUM-185 cells displayed the highest levels of AR followed by MDA-MB-453, MFM-223 and CAL-148 (Additional file 7: Figure S5A). CAL-148 cells contained distinct populations expressing low $\left(A R^{\text {low }}\right)$ and high $\left(A R^{\text {high }}\right)$ levels of $A R$. Sanger sequencing of PIK3CA amplicons from $\mathrm{AR}^{\text {low }}$ and $A R^{\text {high }}$ sorted-cell populations revealed similar frequencies to mutant alleles in each population (Additional file 7: Figure S5B), suggesting that PIK3CA mutation is clonal; whereas there is heterogeneous expression of AR within the cell line.

To determine the relative sensitivity of AR + versus ARTNBC cell lines to PI3K pathway inhibitors, we treated a large panel of cell lines with the pan-PI3K inhibitors GDC-0941 and NVP-BKM120 or with the dual PI3K/ mTOR inhibitors GDC-0980 and NVP-BEZ235. AR + cell lines (indicated in green, Figure 3 ) and other TNBC cell lines containing PIK3CA mutations (indicated in red, Figure 3) were the most sensitive to pharmacological PI3K inhibition, as indicated by low, half-maximal inhibitory concentration (EC50) values. AR + TNBC cells were significantly more sensitive to GDC-0941 (680 nM versus $2044 \mathrm{nM}, P=0.01$ ) and GDC-0980 (66 $\mathrm{nM}$ versus 504 $\mathrm{nM}, P=0.015)$ compared to the other cell lines. In contrast, cell lines with PTEN deficiency, while displaying elevated PI3K pathway signaling, did not uniformly have increased sensitivity to PI3K inhibitors (Figure 3). Although AR + TNBC cells displayed sensitivity to NVPBKM120 and NVP-BEZ235, we chose to highlight the results from GDC-0941 and GDC-0980 in the figures that follow with the results from NVP-BKM120 and NVPBEZ235 included as Additional file 8: supplemental data.

\section{Genetic or pharmacologic inhibition of AR increases sensitivity to PI3K pathway inhibitors}

Since the LAR TNBC cell lines that are sensitive to AR antagonists also harbor highly clonal PIK3CA mutations and are sensitive to PI3K inhibitors [3], we hypothesized that combined targeting of AR and PI3K would more effectively induce growth arrest or apoptosis than either agent alone. To test this hypothesis, we performed both genetic knockdown and pharmacological inhibition of AR with shRNA and CDX, respectively, in the presence and absence of PI3K pathway inhibitors. Immunoblot analysis confirmed decreased AR protein levels in all four AR-expressing TNBC cell lines infected with lentivirus containing two AR-targeting hairpins (shAR-1 and shAR-2) relative to those expressing control, nontargeting shRNA (Figure 4A). Both shRNA expression vectors targeting $A R$ decreased cell viability alone and across increasing doses of GDC-0941 and GDC-0980 (Figure 4B). The most significant difference in viability was observed in cell lines (CAL-148 and MDA-MB453), where the greatest knockdown of AR was achieved. Similar results were obtained with NVP-BKM-120 and NVP-BEZ235, further suggesting that the results observed were specific to inhibition of the PI3K pathway and not off-target drug effects (Additional file 9: Figure S6).

Given that knockdown of AR had an additive antitumor effect with PI3K inhibitors, we pharmacologically inhibited AR using the AR antagonist CDX. Treatment with either GDC-0941 or GDC-0980 decreased proliferation/survival in a dose-dependent manner in AR + TNBC cell lines; the addition of $25 \mu \mathrm{M}$ CDX shifted the survival curves to or below the predicted line of additivity calculated from the effect of each agent alone (Figure 5A). We observed the greatest degree of synergy with the two classes of drugs in cell lines in which CDX was less effective alone (MFM-223) as demonstrated by dose-response curves below the line of additivity (Figure 5A). In contrast, we only observed an additive inhibitory effect when the AR antagonist was used in combination with PI3K inhibitors in cell lines that were more sensitive to single agent CDX (CAL148, MDAMB-453 and SUM185) (Figure 5A).

To verify that the PI3K inhibitors were hitting the target, we performed immunoblot analyses on lysates harvested from control cultures or cells treated alone or in combination with CDX $(25 \mu \mathrm{M})$, GDC-0941 (300 nM) and GDC-0980 (100 nM). Treatment with GDC-0941 and GDC-0980 decreased AKT activity as assessed by levels of phosphorylated AKT (p-S473). As anticipated, GDC-0980 was more effective at decreasing mTOR activity, measured by the decrease in levels of phosphorylated S6 (Figure 5B). In contrast, $\mathrm{CDX}$ treatment alone had minimal effect on either the PI3K/mTOR pathway or AR levels. Interestingly, GDC-0980 treatment alone decreased AR levels and 


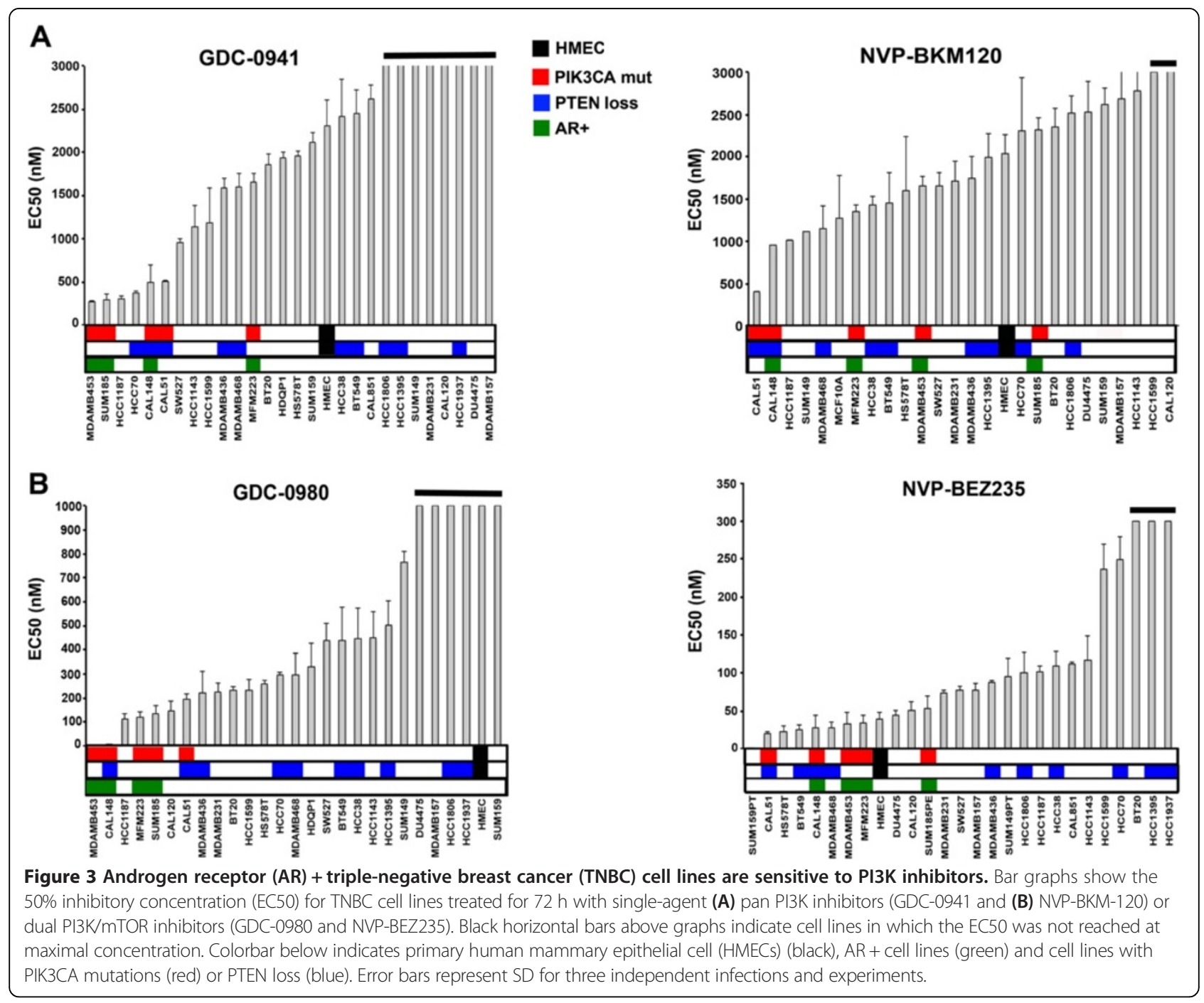

this effect could be enhanced further by the addition of CDX, suggesting that mTOR activity may contribute to elevated AR protein levels (Figure 5B), similar to the signaling observed in prostate cancer [13]. Similar results were obtained with NVP-BKM-120 and NVP-BEZ-235 (Additional file 10: Figure S7).

To determine if the observed decrease in cell number in the AR + TNBC cultures, which were treated with the combination of pathway inhibitors, was attributable to apoptotic cell death, we measured activated caspase-3 and caspase-7 levels after drug treatments (Additional file 11: Figure S8). As a positive control, we prepared lysates from cells treated with adriamycin (ADR, $3 \mu \mathrm{M}$ ) and observed caspase activation at $48 \mathrm{~h}$ (Additional file 11: Figure S8). While CDX treatment alone did not elevate caspase activity, treatment with single agent GDC0941 and GDC-0980 elevated levels of cleaved caspase-3 and -7 with the levels increasing 1.2 to 2 -fold when
PI3K inhibitors were used in combination with CDX (Additional file 11: Figure S8A).

In addition to caspase activity, we quantified the percentage of sub 2 N DNA (sub-G1)-containing cells by FACS analysis (indicative of late stage apoptotic DNA fragmentation). In contrast to the absence of caspase cleavage describe above, CDX treatment alone led to an elevation in the sub- $\mathrm{G}_{1}$ fraction $48 \mathrm{~h}$ after treatment (Additional file 11: Figure S8B). Treatment with GDC0941 or GDC-0980 resulted in higher sub- $\mathrm{G}_{1}$ fraction that increased after combination treatment with CDX (average increase $6.0 \%$ for GDC-0941 and 17.1\% for GDC-0980) (Additional file 11: Figure S8B and C), concordant with the elevated caspase activity observed in Additional file 11: Figure S8A. Therefore, the decreased viability of $\mathrm{AR}+\mathrm{TNBC}$ cell lines treated with a combination of AR and PI3K inhibitors can be, in part, attributed to increased apoptosis. 


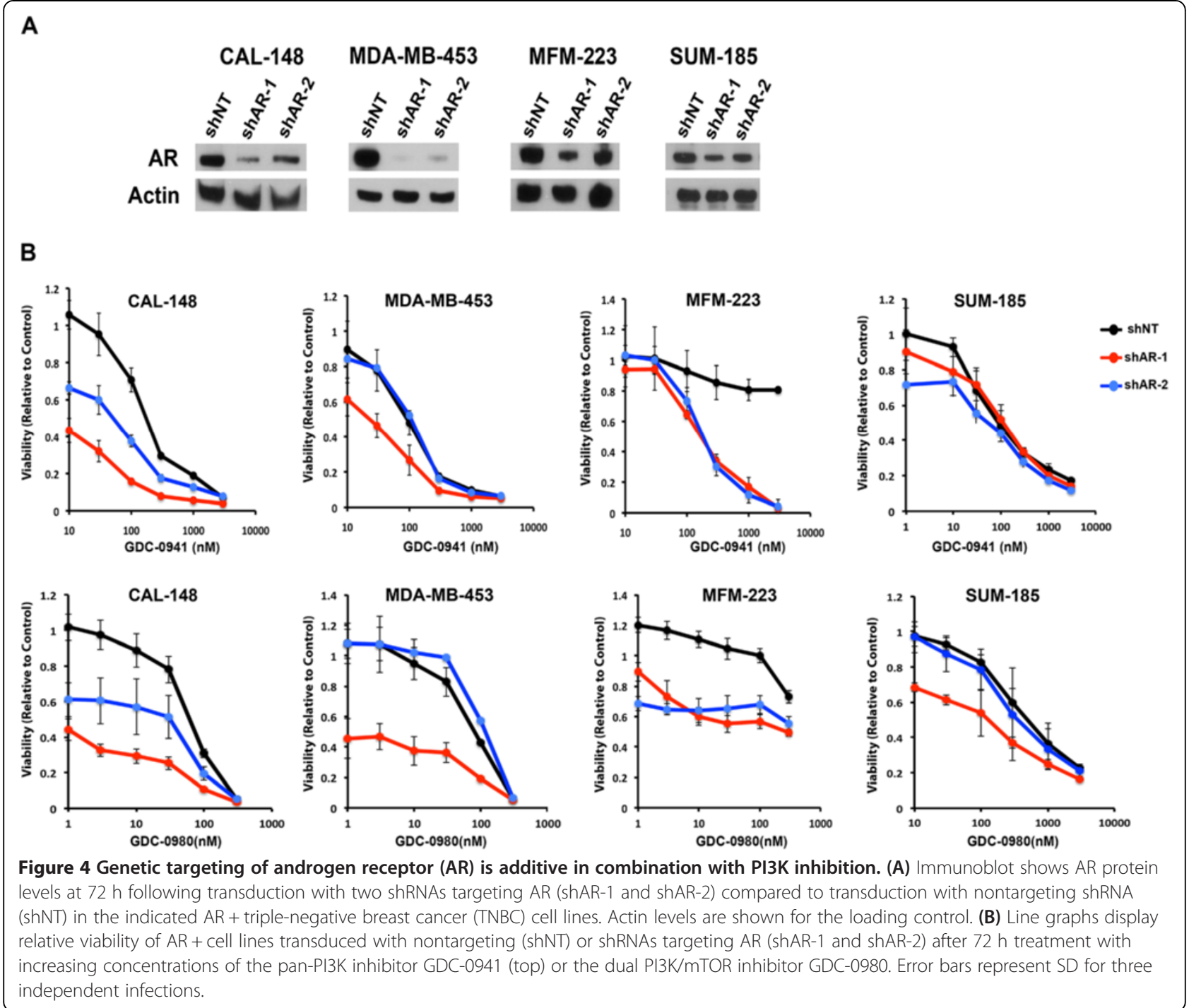

Simultaneous targeting of AR and PI3K signaling decreases viability of AR-expressing cell lines grown in the absence of adhesion

As cell viability and drug sensitivity can be different in 2-dimensional versus 3-dimensional growth conditions, we evaluated combined targeting of AR and PI3K in a forced-suspension assay devoid of matrix. Cell lines grown in suspension were treated with GDC-0941 or GDC0980 alone or in combination with CDX (Figure 6). The addition of CDX was either additive or synergistic with PI3K inhibition (Figure 6B). Similar results were obtained when PI3K was inhibited with NVP-BKM120 or NVP-BEZ235 (Additional file 12: Figure S9). However, when comparing 3-dimensional (Figure 6B) to 2-dimensional growth, we observed that viability was greater by approximately 2 -fold across all doses in the 3-dimensional cultures on average. The results suggest that simultaneous targeting of AR and
PI3K signaling pathways is more effective than targeting either pathway alone in $\mathrm{AR}+\mathrm{TNBC}$ in monolayer or absence of adhesion in a forced suspension assay.

\section{Simultaneous targeting of AR and PI3K signaling} decreases the growth of LAR cell line-derived xenograft tumors in athymic mice

Based on the results from 2-dimensional and 3-dimensional cell culture models, we investigated the effect of combined targeting of $\mathrm{AR}$ and PI3K in vivo in LAR cell line-derived tumor xenografts. We established MDA-MB453 and CAL-148 tumors in athymic nude mice. When tumors reached approximately $50 \mathrm{~mm}^{3}$ in size, the mice were either treated with vehicle, CDX $(100 \mathrm{mg} / \mathrm{kg} / \mathrm{d})$, GDC0941 (50 mg/kg/d), or GDC-0980 (7.5 mg/kg/d) alone or in combinations for 13 to 21 days depending on growth rate of the cell line. Although CDX alone did not 

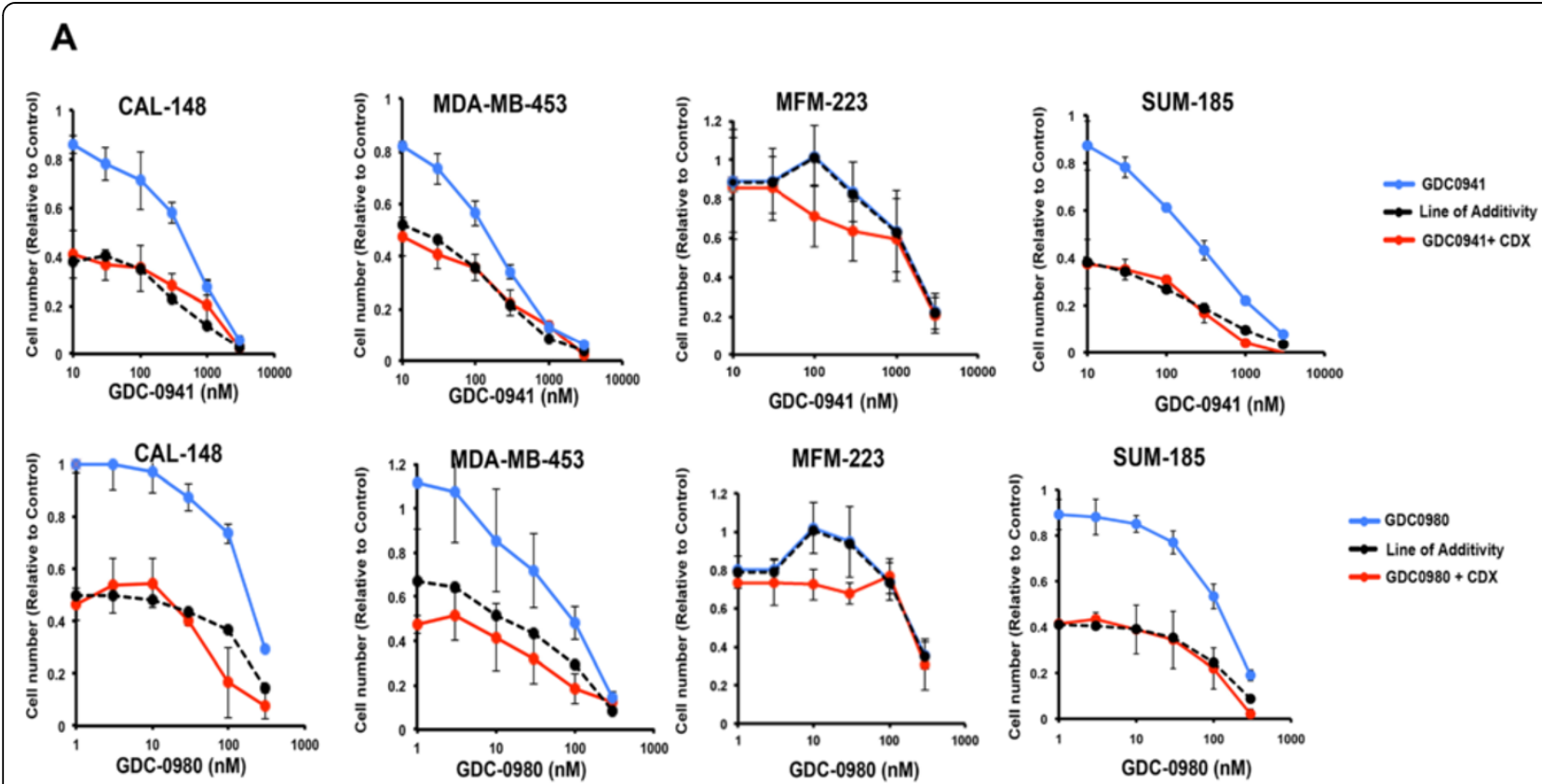

B
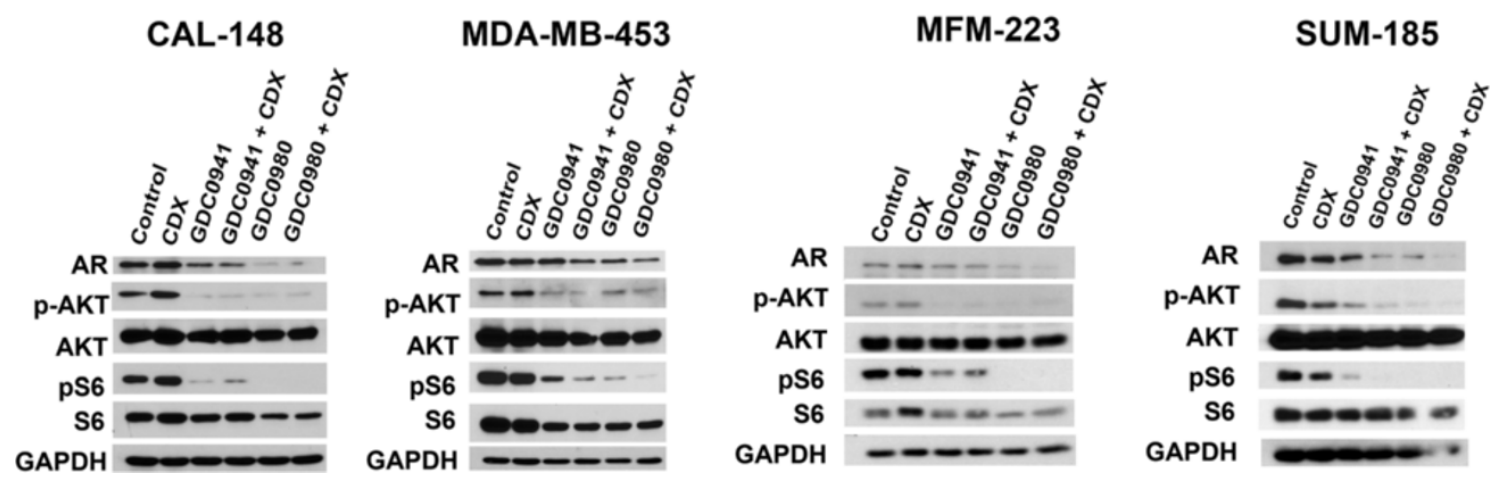

Figure 5 Pharmacological targeting of androgen receptor (AR) with bicalutamide (CDX) is additive in combination with of GDC0941 and GDC0980 in AR + triple-negative breast cancer (TNBC) cell lines. (A) Line graphs show viability of AR + cell lines treated with increasing concentrations of GDC-0941 (top) or GDC-0980 (bottom) alone (blue) or in combination (red) with 25 M CDX. Dashed black line depicts the theoretical line of additivity of both drugs determined from the effect of CDX alone and either GDC-0941 or GDC-0980 alone. Error bars represent SD for three independent experiments. (B) Immunoblots from AR + TNBC cell lines treated with either CDX (25 $\mu$ M), GDC-0941 (300 nM) or GDC0980 $(100 \mathrm{nM})$ as single agents or CDX in combination with either GDC-0941 or GDC-0980 for $48 \mathrm{~h}$ analyzed for AR, p-AKT, AKT, p-S6, S6 and glyceraldehyde3-phosphate dehydrogenase (GAPDH) protein.

decrease tumor size, it significantly slowed growth of both MDA-MD-453 (319 versus $215 \mathrm{~mm}^{3}, P=0.047$ ) and CAL-148 (1394 versus $540 \mathrm{~mm}^{3}, P=0.063$ ) xenograft tumors (Figure 6C). Both single agents GDC-0941 and GDC-0980 significantly reduced tumor growth of MDA-MD-453 $\left(319 \mathrm{~mm}^{3}\right.$ versus $199 \mathrm{~mm}^{3}$ and $57 \mathrm{~mm}^{3}$ respectively, $P=0.0001)$ and CAL-148 $\left(1394 \mathrm{~mm}^{3}\right.$ versus $388 \mathrm{~mm}^{3}$ and $171 \mathrm{~mm}^{3}$ respectively, $P=0.050$ and $P=$ 0.004) (Figure $6 \mathrm{C}$ ). Since the PI3K inhibitors were very effective alone, the added benefit of CDX was difficult to evaluate. However, the combination of CDX with GDC0941 significantly decreased the volume of MDA-MB-453 xenograft tumors $\left(149 \mathrm{~mm}^{3}\right.$ versus $\left.36 \mathrm{~mm}^{3}, P=0.003\right)$ and CDX in combination with GDC0980 significantly decreased growth $\left(171 \mathrm{~mm}^{3}\right.$ versus $\left.97 \mathrm{~mm}^{3,} P=0.041\right)$ of CAL-148 xenograft tumors compared to single agent PI3K inhibition (Figure 6C). GDC-0980 alone or in combination with CDX reduced tumor growth more than GDC-0941, suggesting that concomitant targeting of mTOR provides additional therapeutic benefit or alternatively that the pharmacodynamics of the dosing protocol was superior for GDC-0980.

\section{Discussion}

Herein, we show that activating kinase mutations in PIK3CA are a frequent event in a subset of AR + TNBC 
A
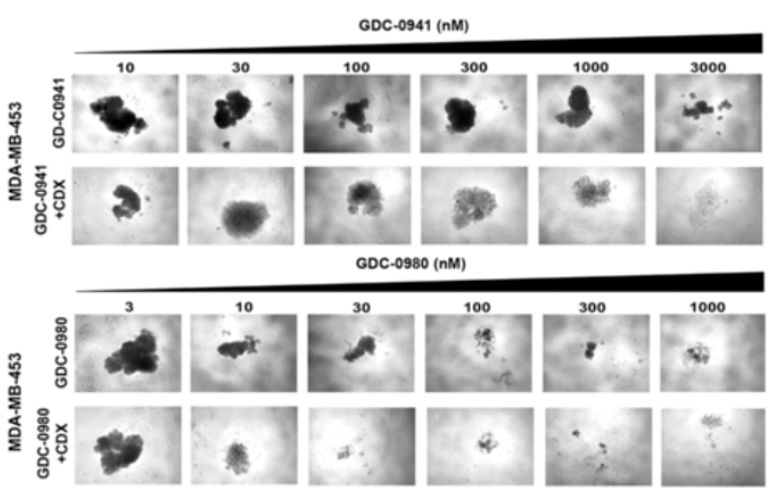

B
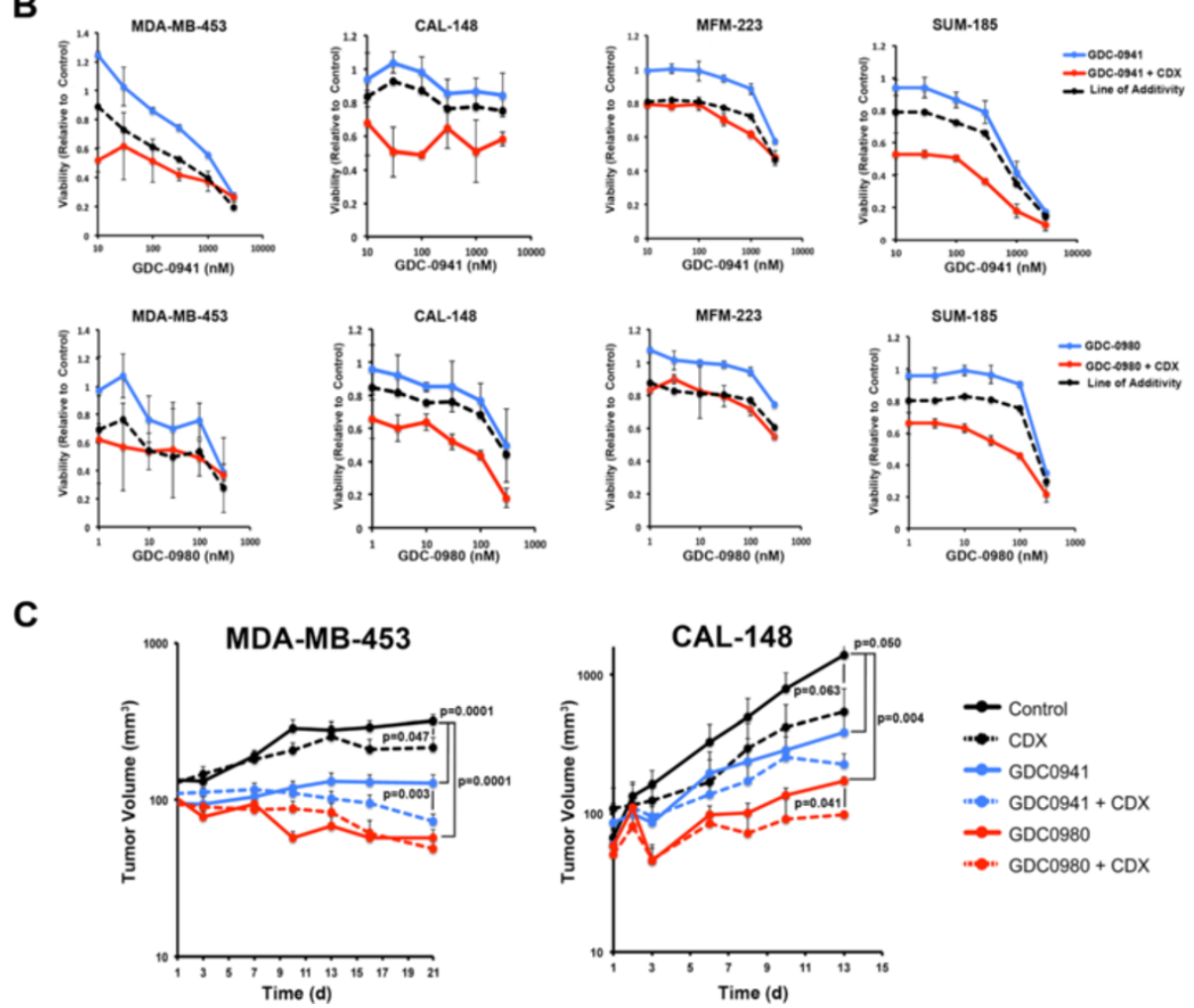

Figure 6 Simultaneous pharmacological targeting of androgen receptor (AR) and PI3K decreases viability of AR + triple-negative breast cancer (TNBC) cell lines grown in 3-dimensional suspension culture or in vivo as xenograft tumors. (A) Bright field images display 3-dimensional cell aggregates of MDA-MB-453 cells treated with increasing doses of GDC-0941 or GDC-0980 in the absence or presence of CDX (25 MM). (B) Line graphs display relative viability of 3-dimensional cell aggregates treated with GDC-0941 or GDC-0980 alone (blue) or in combination (red) with $25 \mu \mathrm{M}$ bicalutamide (CDX). Dashed black line depicts the theoretical line of additivity determined from the effect of CDX alone and either GDC-0941 or GDC-0980 alone. (C) Athymic nude mice bearing established xenograft tumors from AR + TNBC cell lines (MDA-MB-453 and CAL-148) were divided into equal cohorts $(n=8)$ treated with either CDX (100 mg/kg/d, black hashed line), GDC-0941 (100 mg/kg/d, blue line), GDC-0980 $(7.5 \mathrm{mg} / \mathrm{kg} / \mathrm{d}$ red line) or with the combination of CDX and either GDC0-0941 (blue hashed line) or GDC-0980 (red hashed line). Serial-tumor volumes $\left(\mathrm{mm}^{3}\right)$ were measured at the indicated days. Each data point represents mean tumor volume of 16 tumors; error bars represent standard error of the mean.

tumors and cell lines. A link between the PI3K/mTOR pathway and AR signaling was previously established in prostate cancer [14] and PIK3CA mutations are enriched in ER + breast cancer [7]. We demonstrate that not only are PIK3CA mutations predominant in $\mathrm{AR}+\mathrm{TNBC}$, but that they are co-selected with amplification of the PIK3CA locus. Concurrent amplifications and mutations in PIK3CA have previously been reported in uterine serous carcinoma [15], in uterine corpus endometriod cancer (9 of 17 amplified, 53\%), head and neck squamous 
carcinoma (15 of 60 amplified, 25\%), lung squamous carcinoma (10 of 67 amplified, 15\%), and breast cancer (16 of 37 amplified, 43\%) [16,17]. The co-occurrence of amplification and mutation for PIK3CA is far more frequent than for other known oncogenes (BRAF, KRAS, ERBB2), in which mutations and amplifications tend to be mutually exclusive. This finding suggests that a higher level of PIK3CA activity may be needed to overcome a negative regulatory mechanism or that one or more genes in the 3q26 amplicon containing PIK3CA are functionally important.

Mechanistic insights to the evolution of hormonedependent tumors with deregulated PI3K signaling were first provided by Carver et al., who demonstrated that inhibition of AR activates AKT signaling by reducing levels of the AKT phosphatase PHLPP in prostate cancer cells [14]. A recent siRNA screen identified AKT1 as a target that impaired the growth of $\mathrm{AR}+$ prostate cancer cell lines, consistent with the PI3K pathway playing an essential role in survival of AR-dependent cells [18]. Interestingly, while AR has been shown to directly inhibit PTEN transcription in prostate cancer cells [14], AR has been reported to activate PTEN transcription in breast cancer cells [19]. If the latter were the case, elevated PIK3CA protein activity (through mutation and amplification) in AR + TNBC tumors would provide a mechanism to bypass the AR-mediated upregulation of PTEN. Therefore, targeting AR alone could potentially promote survival by reducing PTEN expression and activating the PI3K pathway. Thus, our working hypothesis is that dual targeting of PI3K and AR will provide a synergistic anti-tumor effect.

We and others have shown that approximately 10 to $20 \%$ of TNBCs express AR [3,20]. In addition, Farmer and colleagues have previously identified a distinct gene expression pattern in ER-, AR + breast cancers termed molecular apocrine representing 8 to $14 \%$ of all breast cancers [21]. Clinically, AR-negative patients have a higher likelihood of achieving pathological complete response (pCR) with neoadjuvant chemotherapy than $\mathrm{AR}+$ patients [22]. In addition, Masuda et al. recently completed a retrospective TNBC subtype analysis of gene expression performed on 130 pretreatment biopsies from TNBC patients treated with anthracycline, cytoxan and taxane. While the overall pCR response was $28 \%$, patients with the LAR subtype had a poor response with only $10 \%$ achieving PCR prior to surgery [23]. These findings and the observation that $\mathrm{AR}+\mathrm{TNBC}$ cell lines are relatively resistant to cisplatin [3], suggest TNBC patients with AR + tumors will not receive significant clinical benefit from standard chemotherapy and require novel targeted approaches.

An ongoing clinical trial evaluating CDX as a single agent in metastatic AR+, ER-/PR- breast cancers (NCT00468715/ TBCRC011) has demonstrated some efficacy with a clinical benefit rate of 19\% [24]. While combined inhibition of AR and the PI3K pathway has yet to be carried out in clinical trials, combinations of PI3K inhibitors with the ER downregulator, fulvestrant, are now underway in postmenopausal patients with ER+/HER2- stage IV breast cancer who have progressed on aromatase inhibitors (BELLE-2, NCT01339442) or on the TORC1 inhibitor everolimus (BELLE-3, NCT01633060) [25].

\section{Conclusions}

The results of our study have immediate translational implications for the treatment of AR + TNBC. AR expression is already a robust biomarker with an existing CLIAapproved diagnostic platform that is used in the clinical setting. From published studies, it is apparent that patients with AR + TNBC tumors are likely to demonstrate limited benefit from the current standard-of-care chemotherapy regimens for TNBC $[22,23]$. Thus, our pre-clinical data inform the design of a combination therapy that would be, to our knowledge, the first trial in which TNBC patients are divided based on a biomarker (AR expression) and, as a result, aligned to a targeted investigational combination treatment.

\section{Additional files}

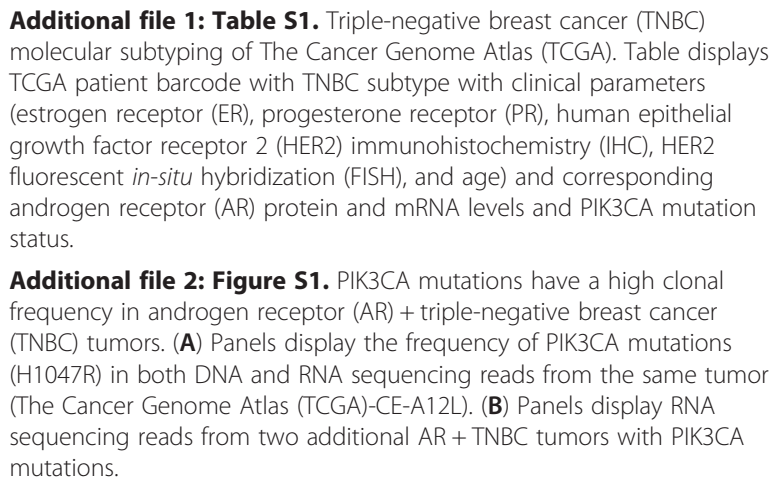

Additional file 2: Figure S1. PIK3CA mutations have a high clonal frequency in androgen receptor (AR) + triple-negative breast cancer (TNBC) tumors. (A) Panels display the frequency of PIK3CA mutations $(\mathrm{H} 1047 \mathrm{R})$ in both DNA and RNA sequencing reads from the same tumor (The Cancer Genome Atlas (TCGA)-CE-A12L). (B) Panels display RNA sequencing reads from two additional $A R+$ TNBC tumors with PIK3CA mutations.

Additional file 3: Figure S2. Frequent concurrent amplification and mutation of PIK3CA in human breast cancers. Image displays 43 tumors with overlapping amplicons of PIK3CA ( $>4$ copies) from The Cancer Genome Atlas (TCGA) breast cohort. Vertical color bars indicate androgen receptor (AR) + triple-negative breast cancer (TNBC) (green) and tumors in which PIK3CA is also mutated (blue).

Additional file 4: Table S2. Linear normalized median-centered $(\log 2)$ reverse-phase protein array (RPPA) values for triple-negative breast cancer (TNBC) cell lines. Table shows median-centered RPPA values for 179 proteins for each of the cell lines, performed in duplicate.

Additional file 5: Figure S3. Triple-negative breast cancer (TNBC) cell lines express both androgen receptor (AR) and p-AKT by immunofluorescence. Immunofluorescent images of MFM-223 (top) and SUM-185 (bottom) co-stained for AR (red), p-AKT(S473) (green) and counterstained with DAPI (blue). Altered cellular morphology is due to cytospin preparation.

Additional file 6: Figure S4. Triple-negative breast cancer (TNBC) cell lines express both androgen receptor (AR) and p-AKT by immunofluorescence. Immunofluorescent images of MDA-MB-453 (top) and CAL-148 (bottom) co-stained for AR (red), p-AKT(S473) (green) and counterstained with DAPI (blue). Arrows indicate a percentage of AR- cells that stain positive for p-AKT.

Additional file 7: Figure S5. PIK3CA mutation in CAL-148 cells is present in both androgen receptor (AR) + and AR- cells sorted by 
fluorescence-activated cell sorting (FACS). (A) FACS scattergrams for AR + triple-negative breast cancer (TNBC) cell lines incubated with alexa fluor 488 secondary antibody alone (control, top panels) or with anti-AR antibody (bottom panels). (B) CAL-148 cells were flow sorted into AR ${ }^{\text {low }}$ (bottom 20\%) and $A R^{\text {high }}$ (top 20\%) populations in which DNA was isolated and PIK3CA evaluated by Sanger sequencing.

\section{Additional file 8: Supplemental methods.}

Additional file 9: Figure S6. shRNA targeting of androgen receptor (AR) is additive in combination with PI3K inhibitors. Line graphs display relative viability of luminal androgen receptor (LAR) cell lines transduced with nontargeting (shNT) or shRNAs targeting AR (shAR-1 and shAR-2) after $72 \mathrm{~h}$ treatment with the pan-PI3K inhibitor NVP-BKM-120 (top) or the dual PI3K/mTOR inhibitor NVP-BEZ235 (bottom). Data represent the average of three replicates.

Additional file 10: Figure S7. Pharmacological targeting of androgen receptor (AR) with bicalutamide (CDX) is additive in combination with PI3K inhibitors in AR + triple-negative breast cancer (TNBC). (A) Line graphs show relative viability of AR-expressing cell lines treated with an increasing concentration of BKM120 (top) or NVP-BEZ235 (bottom) as single agents (blue) or in combination (red) with CDX (25 $\mu \mathrm{M})$. Dashed black line depicts the theoretical line of additivity determined from the effect of CDX alone and either BKM120 or NVP-BEZ235 alone. Error bars represent SD for three independent experiments. (B) Immunoblots from

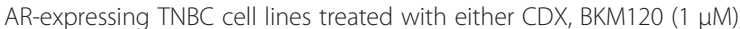
NVP-BEZ235 (100 nM) alone or CDX in combination with either BKM120 or NVP-BEZ235 for $24 \mathrm{~h}$ or $48 \mathrm{~h}$ analyzed for AR, p-AKT, AKT, p-S6, S6 and glyceraldehyde-3-phosphate dehydrogenase (GAPDH) protein.

Additional file 11: Figure S8. Combined inhibition of androgen receptor (AR) and PI3K increases apoptotic cell death in AR + triplenegative breast cancer (TNBC) cell lines. (A) Bar graphs display relative caspase $3 / 7$ activity (RLU) normalized to viable cell number $48 \mathrm{~h}$ after treatment with vehicle, positive control ( $3 \mu \mathrm{M}$ ADR), bicalutamide (CDX) $(50 \mu \mathrm{M}), \mathrm{GDC}-0941(3 \mu \mathrm{M})$ or GDC-0980 $(1 \mu \mathrm{M})$ as single agents or in combination with CDX. Error bars represent SD for three independent experiments. (B) Representative cell cycle histograms of the MDA-MB-453 cell line treated with similar conditions as described above. (C) Bar graphs indicate percentages of sub- $G_{1} D N A$, indicative of late-stage apoptotic DNA fragmentation.

Additional file 12: Figure S9. Simultaneous targeting of androgen receptor (AR) and PI3K decreases viability of AR + triple-negative breast cancer (TNBC) cell lines grown in a 3-D forced suspension assay. Line graphs display relative viability of 3-D cell aggregates treated with BKM120 (top) or NVP-BEZ235 (bottom) as single agents (blue) or in combination (red) with CDX (25 $\mu \mathrm{M})$. Dashed black line depicts the theoretical line of additivity determined from the effect of bicalutamide (CDX) alone and either BKM120 or NVP-BEZ235 alone. Error bars represent SD for three independent experiments.

\begin{abstract}
Abbreviations
ADR: adriamycin; AR+: androgen receptor-positive; BL1 and BL2: basal-like; bp: base pair(s); CAP/ASCO: College of American Pathologists/American Society of Clinical Oncology; CDX: bicalutamide; CS: charcoal-stripped; DAB: 3,3-diaminobenzidine; DHT: dihydrotestosterone; EC50: half-maximal inhibitory concentration; ER: estrogen receptor; FACS: fluorescence-activated cell sorting; FFPE: formalin-fixed paraffin-embedded; FISH: fluorescent in-situ hybridization; GAPDH: glyceraldehyde-3-phosphate dehydrogenase; GE: gene expression; HER2: human epithelial growth factor receptor 2; HMEC: human mammary epithelial cell; IM: immunomodulatory; LAR: luminal androgen receptor; M: mesenchymal; MCT: carboxymethyl cellulose; MSL: mesenchymal stem-like; pCR: pathological complete response; PI3K: phosphoinositide 3-kinase; PIK3CA: phosphatidylinositol-4,5-bisphosphate 3-kinase catalytic subunit alpha; PR: progesterone receptor; RPPA: reverse phase protein array; TCGA: The Cancer Genome Atlas; TNBC: triple-negative breast cancer.
\end{abstract}

\section{Competing interests}

The authors declare that they have no competing interests.

\section{Authors' contributions}

Conception and design: BDL, JAB, and JAP. Development of methodology: $B D L, J A B, J M S, C S P, L T, K C J$, and JAP. Acquisition of data (provided tissue, acquired and managed patients, provided facilities, et cetera): JMB, HG, CLA, GBM, and MES. Analysis and interpretation of data (for example, statistical analysis, biostatistics, computational analysis): BDL, JAB, XC, and JAP. Writing, review, and/or revision of the manuscript: BDL, JAB, JMS, CSP, JMB, HG, CLA, GBM, MES, and JAP. Administrative, technical, or material support (that is, reporting or organizing data, constructing databases): KCJ and BDL. Study supervision: BDL and JAP. All authors have read and approved the final version of this manuscript.

\section{Acknowledgements}

We thank members of the Pietenpol laboratory for critical review of the manuscript. This research was supported by National Institutes of Health Grants: CA95131 (Specialized Program of Research Excellence in Breast Cancer); CA148375; CA105436 and CA070856 (to JA Pietenpol); CA68485 (core services); CA009385 (JAB); Komen for the Cure Foundation Grant SAC110030 (to JA Pietenpol) and KG262005 (to BD Lehmann), American Cancer Society Grant PF-10-226-01-TBG (to BD Lehmann) and a Susan G Komen Promise Grant KG081694 (to GBM).

\section{Author details}

${ }^{1}$ Department of Biochemistry, Vanderbilt-Ingram Cancer Center, Vanderbilt University School of Medicine, Preston Research Building, 2200 Pierce Avenue, Nashville, TN 37232, USA. 'Department of Biostatistics, Vanderbilt-Ingram Cancer Center, Vanderbilt University School of Medicine, Preston Research Building, 2200 Pierce Avenue, Nashville, TN 37232, USA. ${ }^{3}$ Department of Medicine, Vanderbilt-Ingram Cancer Center, Vanderbilt University School of Medicine, Preston Research Building, 2200 Pierce Avenue, Nashville, TN 37232, USA. Instituto Nacional de Enfermedades Neoplásicas, Av. Angamos Este 2520, Surquillo, Lima 34, Peru. ${ }^{5}$ Department of Systems Biology, The University of Texas MD Anderson Cancer Center, 1400 Pressler Street, Unit 1484, Houston, TX 77030, USA. ${ }^{6}$ Department of Pathology Vanderbilt-Ingram Cancer Center, Vanderbilt University School of Medicine, Preston Research Building, 2200 Pierce Avenue, Nashville, TN 37232, USA. Vanderbilt-Ingram Cancer Center, 652 Preston Research Building, Nashville, TN 37232, USA.

Received: 10 February 2014 Accepted: 4 July 2014

Published: 8 August 2014

\section{References}

1. Lin NU, Vanderplas A, Hughes ME, Theriault RL, Edge SB, Wong YN, Blayney DW, Niland JC, Winer EP, Weeks JC: Clinicopathologic features, patterns of recurrence, and survival among women with triple-negative breast cancer in the National Comprehensive Cancer Network. Cancer 2012, 118:5463-5472.

2. Dent R, Trudeau M, Pritchard KI, Hanna WM, Kahn HK, Sawka CA, Lickley LA, Rawlinson E, Sun P, Narod SA: Triple-negative breast cancer: clinical features and patterns of recurrence. Clin Cancer Res 2007, 13:4429-4434.

3. Lehmann BD, Bauer JA, Chen X, Sanders ME, Chakravarthy AB, Shyr Y, Pietenpol JA: Identification of human triple-negative breast cancer subtypes and preclinical models for selection of targeted therapies. J Clin Invest 2011, 121:2750-2767.

4. Keam B, Im SA, Kim HJ, Oh DY, Kim JH, Lee SH, Chie EK, Han W, Kim DW, Moon WK, Kim TY, Park IA, Noh DY, Heo DS, Ha SW, Bang YJ: Prognostic impact of clinicopathologic parameters in stage II/III breast cancer treated with neoadjuvant docetaxel and doxorubicin chemotherapy: paradoxical features of the triple negative breast cancer. BMC Cancer 2007, 7:203.

5. Shah SP, Roth A, Goya R, Oloumi A, Ha G, Zhao Y, Turashvili G, Ding J, Tse K, Haffari G, Bashashati A, Prentice LM, Khattra J, Burleigh A, Yap D, Bernard V, McPherson A, Shumansky K, Crisan A, Giuliany R, Heravi-Moussavi A, Rosner J, Lai D, Birol I, Varhol R, Tam A, Dhalla N, Zeng T, Ma K, Chan SK, et al: The clonal and mutational evolution spectrum of primary triple-negative breast cancers. Nature 2012, 486:395-399.

6. Cancer Genome Atlas Network: Comprehensive molecular portraits of human breast tumours. Nature 2012, 490:61-70.

7. Saal LH, Holm K, Maurer M, Memeo L, Su T, Wang X, Yu JS, Malmström P-O, Mansukhani M, Enoksson J, Hibshoosh H, Borg A, Parsons R: PIK3CA mutations 
correlate with hormone receptors, node metastasis, and ERBB2, and are mutually exclusive with PTEN loss in human breast carcinoma. Cancer Res 2005, 65:2554-2559.

8. Gonzalez-Angulo AM, Stemke-Hale K, Palla SL, Carey M, Agarwal R, Meric-Berstam F, Traina TA, Hudis C, Hortobagyi GN, Gerald WL, Mills GB, Hennessy BT: Androgen receptor levels and association with PIK3CA mutations and prognosis in breast cancer. Clin Cancer Res 2009, 15:2472-2478

9. Hammond MEH, Hayes DF, Dowsett M, Allred DC, Hagerty KL, Badve S, Fitzgibbons PL, Francis G, Goldstein NS, Hayes M, Hicks DG, Lester S, Love R, Mangu PB, McShane L, Miller K, Osborne CK, Paik S, Perlmutter J, Rhodes A, Sasano H, Schwartz JN, Sweep FCG, Taube S, Torlakovic EE, Valenstein P, Viale G, Visscher D, Wheeler T, Williams RB, et al: American Society of Clinical Oncology/College of American Pathologists guideline recommendations for immunohistochemical testing of estrogen and progesterone receptors in breast cancer. Arch Pathol 2010, 134:907-922.

10. Wolff ACA, Hammond MEHM, Schwartz JNJ, Hagerty KLK, Allred DCD, Cote RJR, Dowsett MM, Fitzgibbons PLP, Hanna WMW, Langer AA, McShane LML, Paik SS, Pegram MDM, Perez EAE, Press MFM, Rhodes AA, Sturgeon CC, Taube SES, Tubbs RR, Vance GHG, van de Vijver MM, Wheeler TMT, Hayes DFD: American Society of Clinical Oncology/College of American Pathologists guideline recommendations for human epidermal growth factor receptor 2 testing in breast cancer. Audio, Transactions of the IRE Professional Group on 2007, 25:118-143.

11. Chen X, Li J, Gray WH, Lehmann BD, Bauer JA, Shyr Y, Pietenpol JA: TNBCtype: A Subtyping Tool for Triple-Negative Breast Cancer. Cancer Inform 2012, 11:147-156.

12. Zhang L, Zhang L, Wei Q, Wei Q, Mao L, Mao L, Liu W, Liu W, Mills GB, Mills GB, Coombes K, Coombes K: Serial dilution curve: a new method for analysis of reverse phase protein array data. Bioinformatics 2009, 25:650-654.

13. Lin $\mathrm{H}-\mathrm{K}$, Hu $\mathrm{Y}-\mathrm{C}$, Yang $\mathrm{L}$, Altuwaiji $\mathrm{S}$, Chen $\mathrm{Y}-\mathrm{T}$, Kang $\mathrm{H}-\mathrm{Y}$, Chang $\mathrm{C}$ Suppression versus induction of androgen receptor functions by the phosphatidylinositol 3-kinase/Akt pathway in prostate cancer LNCaP cells with different passage numbers. J Biol Chem 2003, 278:50902-50907.

14. Carver BS, Chapinski C, Wongvipat J, Hieronymus H, Chen Y, Chandarlapaty S, Arora VK, Le C, Koutcher J, Scher H, Scardino PT, Rosen N, Sawyers CL: Reciprocal feedback regulation of $\mathrm{PI} 3 \mathrm{~K}$ and androgen receptor signaling in PTEN-deficient prostate cancer. Cancer Cell 2011, 19:575-586.

15. Kuhn E, Wu R-C, Guan B, Wu G, Zhang J, Wang Y, Song L, Yuan X, Wei L, Roden RBS, Kuo K-T, Nakayama K, Clarke B, Shaw P, Olvera N, Kurman RJ, Levine DA, Wang T-L, Shih I-M: Identification of molecular pathway aberrations in uterine serous carcinoma by genome-wide analyses. J Natl Cancer Inst 2012, 104:1503-1513.

16. Gao J, Aksoy BA, Dogrusoz U, Dresdner G, Gross B, Sumer SO, Sun Y Jacobsen A, Sinha R, Larsson E, Cerami E, Sander C, Schultz N: Integrative Analysis of Complex Cancer Genomics and Clinical Profiles Using the cBioPortal. Sci Signal 2013, 6:pl1-pl1.

17. Cerami E, Gao J, Dogrusoz U, Gross BE, Sumer SO, Aksoy BA, Jacobsen A, Byrne CJ, Heuer ML, Larsson E, Antipin Y, Reva B, Goldberg AP, Sander C, Schultz N: The cBio cancer genomics portal: an open platform for exploring multidimensional cancer genomics data. Cancer Discovery 2012, 2:401-404

18. Dahlman KB, Parker JS, Shamu T, Hieronymus H, Chapinski C, Carver B, Chang K, Hannon GJ, Sawyers CL: Modulators of prostate cancer cell proliferation and viability identified by short-hairpin RNA library screening. PLOS ONE 2012, 7:e34414.

19. Wang $Y$, Romigh $T$, He $X$, Tan MH, Orloff MS, Silverman RH, Heston WD, Eng C: Differential regulation of PTEN expression by androgen receptor in prostate and breast cancers. Oncogene 2011, 30:4327-4338.

20. Gucalp A, Traina TA: Triple-negative breast cancer: role of the androgen receptor. Cancer J 2010, 16:62-65.

21. Farmer PP, Bonnefoi HH, Becette $W$, Tubiana-Hulin MM, Fumoleau PP, Larsimont DD, Macgrogan GG, Bergh JJ, Cameron DD, Goldstein DD, Duss SS, Nicoulaz A-LA, Brisken CC, Fiche MM, Delorenzi MM, Iggo RR: Identification of molecular apocrine breast tumours by microarray analysis. Oncogene 2005, 24:4660-4671.

22. Loibl S, Muller BM, Minckwitz von G, Schwabe M, Roller M, Darb-Esfahani S, Ataseven B, Bois du A, Fissler-Eckhoff A, Gerber B, Kulmer U, Alles JU, Mehta $K$, Denkert C: Androgen receptor expression in primary breast cancer and its predictive and prognostic value in patients treated with neoadjuvant chemotherapy. Breast Cancer Res Treat 2011, 130:477-487.

23. Masuda H, Baggerly KA, Wang Y, Ya Z, Gonzalez-Angulo AM, MericBernstam F, Valero V, Lehmann BD, Pietenpol JA, Hortobagyi GN, Symmans WF, Ueno NT: Differential response to neoadjuvant chemotherapy among 7 triple-negative breast cancer molecular subtypes. Clin Canc Res 2013, 19:5533-5540

24. Gucalp A, Tolaney S, Isakoff SJ, Ingle JN, Liu MC, Carey LA, Blackwell KL, Rugo H, Nabell L, Forero-Torres A, Stearns V, Doane AS, Danso M, Moynahan ME, Momen LF, Gonzalez JM, Akhtar A, Giri D, Patil S, Feigin KN, Hudis CA, Traina TA: Phase II Trial of Bicalutamide in Patients with Androgen Receptor Positive, Hormone Receptor Negative Metastatic Breast Cancer. Clin Canc Res 2013, 19:5505-5512.

25. Iwata H, Baselga J, Campone M, Arteaga CL, Cortes J, Jonat W, De Laurentiis M, Ciruelos E, Janni W, Bachelot T, Lonning PE, ORegan R, Di Tomaso E, Bharani-Dharan B, Duval V, Lau H, Germa C, Urban P, Di Leo A: Ph III randomized studies of the oral pan-PI3K inhibitor buparlisib (BKM120) with fulvestrant in postmenopausal women with HR+/HER2- locally advanced or metastatic breast cancer $(B C)$ after aromatase inhibitor (Al; BELLE-2) or Al and mTOR inhibitor (BELLE-3) treatment. J Clin Oncol 2013, 31, TPS650.

doi:10.1186/s13058-014-0406-x

Cite this article as: Lehmann et al.: PIK3CA mutations in androgen receptor-positive triple negative breast cancer confer sensitivity to the combination of $\mathrm{PI} 3 \mathrm{~K}$ and androgen receptor inhibitors. Breast Cancer Research 2014 16:406.

\section{Submit your next manuscript to BioMed Central and take full advantage of:}

- Convenient online submission

- Thorough peer review

- No space constraints or color figure charges

- Immediate publication on acceptance

- Inclusion in PubMed, CAS, Scopus and Google Scholar

- Research which is freely available for redistribution 